\title{
Structure of ${ }^{12}$ Be: Intruder $d$-Wave Strength at $N=8$
}

\author{
S. D. Pain, ${ }^{1, *}$ W. N. Catford, ${ }^{1,2}$ N. A. Orr, ${ }^{2}$ J. C. Angélique, ${ }^{2}$ N. I. Ashwood,${ }^{3}$ V. Bouchat,${ }^{4}$ N. M. Clarke, ${ }^{3}$ N. Curtis,${ }^{3}$ \\ M. Freer, ${ }^{3}$ B. R. Fulton, ${ }^{5}$ F. Hanappe, ${ }^{4}$ M. Labiche, ${ }^{6}$ J. L. Lecouey, ${ }^{2}$ R. C. Lemmon, ${ }^{7}$ D. Mahboub,${ }^{1}$ A. Ninane, ${ }^{8}$ \\ G. Normand, ${ }^{2}$ N. Soić, ${ }^{3, \dagger}$ L. Stuttge, ${ }^{9}$ C. N. Timis, ${ }^{1}$ J. A. Tostevin, ${ }^{1}$ J. S. Winfield, ${ }^{1,2}$ and V. Ziman ${ }^{3}$ \\ ${ }^{1}$ Department of Physics, University of Surrey, Guildford, GU2 7XH, United Kingdom \\ ${ }^{2}$ Laboratoire de Physique Corpusculaire, ENSICAEN et Université de Caen, IN2P3-CNRS, 14050 Caen Cedex, France \\ ${ }^{3}$ School of Physics and Astronomy, University of Birmingham, Edgbaston, Birmingham, B15 2TT, United Kingdom \\ ${ }^{4}$ Université Libre de Bruxelles, CP226, B-1050 Bruxelles, Belgium \\ ${ }^{5}$ Department of Physics, University of York, Heslington, York, YO10 5DD, United Kingdom \\ ${ }^{6}$ Electronic Engineering and Physics, University of Paisley, High Street, Paisley, Scotland, PA1 2BE, United Kingdom \\ ${ }^{7}$ CCLRC Daresbury Laboratory, Daresbury, Warrington, Cheshire, WA4 4AD, United Kingdom \\ ${ }^{8}$ Insitut de Physique Nucléare, Université Catholique de Louvain, Louvain-la-Neuve, Belgium \\ ${ }^{9}$ Institut de Recherche Subatomique, IN2P3-CNRS/Université de Louis Pasteur, BP28, 67037 Strasbourg Cedex, France
}

(Received 26 October 2005; published 27 January 2006)

\begin{abstract}
The breaking of the $N=8$ shell-model magic number in the ${ }^{12} \mathrm{Be}$ ground state has been determined to include significant occupancy of the intruder $d$-wave orbital. This is in marked contrast with all other $N=8$ isotones, both more and less exotic than ${ }^{12} \mathrm{Be}$. The occupancies of the $0 \hbar \omega \nu p_{1 / 2}$ orbital and the $1 \hbar \omega, \nu d_{5 / 2}$ intruder orbital were deduced from a measurement of neutron removal from a high-energy ${ }^{12} \mathrm{Be}$ beam leading to bound and unbound states in ${ }^{11} \mathrm{Be}$.
\end{abstract}

DOI: 10.1103/PhysRevLett.96.032502

A principal aim of present day nuclear structure research is to understand the evolution of shell structure with increasing asymmetry in the neutron-to-proton ratio. In this context the $N=8$ isotonic chain, which spans from ${ }^{22} \mathrm{Si}$ via the doubly magic $N=Z{ }^{16} \mathrm{O}$ to the two-neutron halo system ${ }^{11} \mathrm{Li}$ and the unbound ${ }^{10} \mathrm{He}$, is of considerable interest. Indeed, the $N=8$ shell closure, that is clearly evident close to stability, disappears amongst the lightest of these nuclei. In particular, the halo structure of ${ }^{11} \mathrm{Li}$ is enhanced by a strong $\nu\left(1 s_{1 / 2}\right)^{2}$ intruder valence neutron configuration [1]. Similarly, recent experiments [2-5] have confirmed earlier work [6-9] in which it was concluded that the ${ }^{12} \mathrm{Be}$ ground state is formed from both the "normal" closed shell $\nu\left(0 p_{1 / 2}\right)^{2}$ valence configuration and the intruder $\nu(1 s 0 d)^{2}$ configurations, with a ${ }^{10} \mathrm{Be}($ g.s. $)$ core. The factors producing these intruder configurations appear to include a reduction in the $p$-sd shell gap as the dripline is approached, an increase in the monopole pairing energy, and deformation [10]. The deformation is also believed to be related to the tendency towards alpha-particle clustering [11] in the Be isotopes.

Whereas high-energy single-neutron removal (or "knockout") from ${ }^{12} \mathrm{Be}$ has provided direct evidence for configuration mixing involving the $\nu\left(1 s_{1 / 2}\right)^{2}$ and $\nu\left(0 p_{1 / 2}\right)^{2}$ valence neutron configurations in the ground-state [3], model predictions indicate that a substantial $\nu\left(0 d_{5 / 2}\right)^{2}$ admixture $(\sim 30 \%-50 \%)$ should also be present $[10,12-$ 15]. In the experiment of Navin et al., ${ }^{11}$ Be core fragments in either the $J^{\pi}=1 / 2^{+}$ground state or the bound $320 \mathrm{keV}$ $1 / 2^{-}$excited state were detected [3]. These states have large overlaps with the pure single-particle states $\nu\left(1 s_{1 / 2}\right)$ and $\nu\left(0 p_{1 / 2}\right)$, respectively [16,17]. The measurement of Ref. [3] was, however, not sensitive to the $\nu\left(0 d_{5 / 2}\right)^{2}$ com-
PACS numbers: 25.60.Dz, 21.60.Cs, 24.10. $-\mathrm{i}, 27.20 .+\mathrm{n}$

ponent as the removal of a $0 d_{5 / 2}$ neutron leaves ${ }^{11} \mathrm{Be}$ in the $\nu\left(0 d_{5 / 2}\right) \quad$ single-particle state $\left(E_{x}=1.78 \mathrm{MeV}, \Gamma=\right.$ $100 \mathrm{keV}$ ) [16,18], which is unbound to neutron emission and decays to ${ }^{10} \mathrm{Be}($ g.s. $)+n$. It is thus necessary to design an experiment to detect both the ${ }^{10} \mathrm{Be}$ fragment and the neutron and then to reconstruct their relative decay energy from the measured momenta. This was the approach adopted in the present work. Further, to assist comparisons with theory and the earlier work, the ability to detect ${ }^{11} \mathrm{Be}$ in the first excited state (via the $320 \mathrm{keV} \gamma$ ray) was included.

A secondary beam of ${ }^{12} \mathrm{Be}(\sim 5000 \mathrm{pps})$ was prepared using the LISE3 spectrometer at GANIL from the reaction of a $63 \mathrm{MeV} / A^{18} \mathrm{O}$ beam on ${ }^{9} \mathrm{Be}$. The average ${ }^{12} \mathrm{Be}$ beam energy was $39.3 \mathrm{MeV} / A$ at the center of the carbon secondary reaction target $\left(183 \mathrm{mg} / \mathrm{cm}^{2}\right)$. The beam purity was $95 \%$, with the remaining $5 \%$ being ${ }^{6} \mathrm{He}$ and ${ }^{15} \mathrm{~B}$. Owing to the poor emittance of the secondary beam, the spot size on target was $\sim 10 \mathrm{~mm}$ diameter and the incident ions were tracked event by event using two positionsensitive drift chambers located upstream of the carbon target. The point of impact was thus determined to within $\lesssim 1 \mathrm{~mm}$ at the target. The measured time-of-flight through the LISE spectrometer allowed ${ }^{12} \mathrm{Be}$ ions to be selected uniquely event by event and provided a measure of the energy, with a resolution of $1.6 \%$ (FWHM).

A telescope subtending $\pm 9^{\circ}$ in the horizontal $(x)$ and vertical $(y)$ planes recorded charged particles emerging from the carbon target close to $0^{\circ}$, including the unreacted beam. This was composed of two $50 \times 50 \mathrm{~mm}^{2} 500 \mu \mathrm{m}$ thick Si strip detectors to measure energy loss $\left(\Delta E_{1,2}\right)$, followed by a $4 \times 4$ array of 16 CsI stopping detectors (E) $25 \mathrm{~mm}$ thick [19]. The telescope was calibrated using 
"cocktail" beams containing all relevant isotopes of $\mathrm{Be}$, at several spectrometer settings to span the energies of interest. Using $\Delta E_{1,2}$ and $E$, all observed isotopes of $\mathrm{H}, \mathrm{He}, \mathrm{Li}$, $\mathrm{Be}$, and $\mathrm{B}$ were clearly resolved. The Si detectors provided $x$ and $y$ position measurement which, combined with the drift chamber data, determined the scattering angle with a resolution $0.7^{\circ}$ (FWHM).

Coincident $\gamma$ rays were recorded using $4 \mathrm{NaI}$ detectors mounted around the target at angles of $\pm 45^{\circ}$ and $\pm 110^{\circ}$ to the beam, with a total absolute photopeak efficiency of $3.5 \%$ for detection of Doppler-shifted $320 \mathrm{keV} \gamma$ rays.

Neutrons were detected using the DEMON array of 91 liquid scintillator modules [20] located between $2.4 \mathrm{~m}$ and $6.3 \mathrm{~m}$ downstream of the carbon target and spanning angles out to $32^{\circ}$ [21]. Neutrons were distinguished from $\gamma$ rays using standard pulse-shape discrimination and their energies $\left(E_{n}\right)$ were derived via the time of flight with a resolution $\sim 5 \%$. Neutrons with $E_{n} \leq 15 \mathrm{MeV}$, originating from the target, were excluded in the analysis.

The background arising from ${ }^{12} \mathrm{Be}$ beam particles that reacted in the telescope was determined by acquiring data with no target, with the beam energy lowered to account for the energy loss in the target [21]. Such events gave degraded energy signals, and could be misidentified as ${ }^{11} \mathrm{Be}$ or ${ }^{10} \mathrm{Be}$. This background precluded an accurate measurement of the yield to the ${ }^{11} \mathrm{Be}$ ground state [22]. For the excited states, coincident detection of a $\gamma$ ray or neutron reduced substantially the background (to $50 \%$ and $60 \%$ of the target-in data, respectively).

The background-subtracted, Doppler-corrected $\gamma$-ray spectrum, in coincidence with ${ }^{11} \mathrm{Be}$ ions in the telescope, is shown in Fig. 1. The cross section for production of the $1 / 2^{-}$state in ${ }^{11} \mathrm{Be}$ (see Table I) was extracted after accounting for the experimentally measured $\gamma$-ray detector efficiencies, attenuation in the target, and the relativistic focussing of $\gamma$ rays in the laboratory frame $(\beta \simeq 0.28)$. The cross section measured here at $39.3 \mathrm{MeV} /$ nucleon is compatible with the value measured previously at $78 \mathrm{MeV} /$ nucleon with a Be target [3], as interpreted below using an eikonal reaction model. The longitudinal momentum distribution of the ${ }^{11} \mathrm{Be}^{*}\left(1 / 2^{-}\right)$fragments was also measured, giving a FWHM of $137(21) \mathrm{MeV} / c$, in agreement with $\sim 150 \mathrm{MeV} / c$ estimated from Ref. [3].

The measured momenta of coincident ${ }^{10} \mathrm{Be}$ ions and neutrons were used to kinematically reconstruct unbound states in ${ }^{11} \mathrm{Be}$. The procedure was verified [22] by reconstructing the well-known ground-state resonance of ${ }^{7} \mathrm{He}$ from ${ }^{6} \mathrm{He}+n$ coincidences. The relative energy $\left(E_{\text {rel }}\right)$ spectrum for ${ }^{10} \mathrm{Be}+n$, after background subtraction, is shown in Fig. 2, which is corrected for the intrinsic efficiency of the DEMON detectors but not for the geometrical acceptance. A peak is clearly evident at $\sim 1.3 \mathrm{MeV}$, corresponding to the decay of the $1.78 \mathrm{MeV}\left(5 / 2^{+}\right)$state in ${ }^{11} \mathrm{Be}$. There is also another peak apparent near $2.2 \mathrm{MeV}$, corresponding to decay of the $2.69 \mathrm{MeV}\left(3 / 2^{-}\right)$state [23].

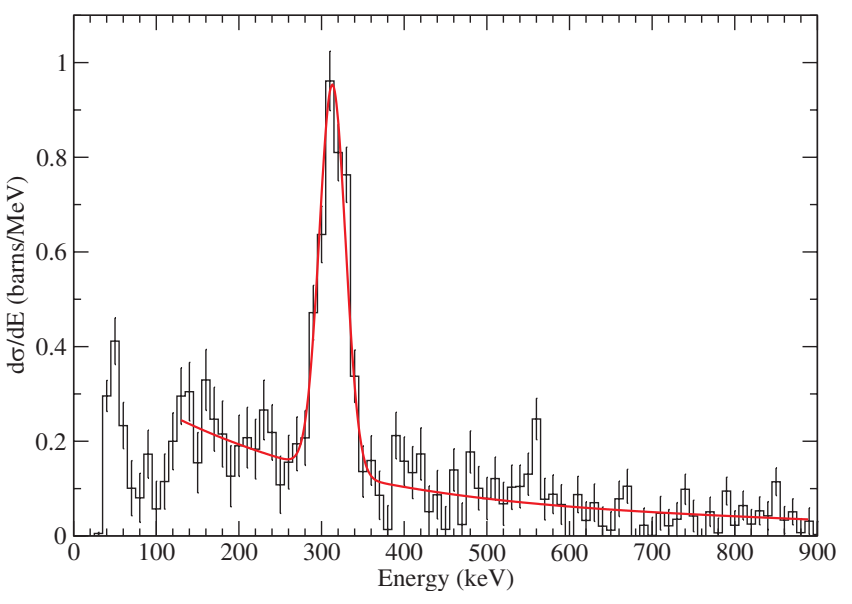

FIG. 1 (color online). Background-subtracted, Dopplercorrected $\gamma$ ray energy spectrum, in coincidence with ${ }^{11} \mathrm{Be}$ fragments following the reaction of ${ }^{12} \mathrm{Be}(39.3 \mathrm{MeV} /$ nucleon $)$ on a carbon target. The full line is the result of a Gaussian fit, with an exponential background. From this, the cross section shown in Table I was deduced for ${ }^{11} \mathrm{Be}$ in the first excited $\left(1 / 2^{-}\right)$ state.

The very narrow peak near threshold is compatible with decay from a state at $\sim 4.0 \mathrm{MeV}$ in ${ }^{11} \mathrm{Be}$ to the first $2^{+}$state in ${ }^{10} \mathrm{Be}$ at $3.37 \mathrm{MeV}$. The ground-state branch of this decay corresponds to the peak at $E_{\text {rel }} \sim 3.5 \mathrm{MeV}$; its inclusion improves the fit, but the magnitude of neither peak is well defined by the data. However, good candidates exist for such a state in ${ }^{11} \mathrm{Be}[18,24]$.

The detection efficiency for neutrons from ${ }^{11} \mathrm{Be}^{*}$ decay is determined partly by their laboratory angular distribution, which depends on the decay energy to ${ }^{10} \mathrm{Be}+n$ and the spread in momentum induced by the initial neutron removal from ${ }^{12} \mathrm{Be}$. Detailed simulations were performed [22], including the effects of the geometric acceptance of the neutron detector array, the energy and angular straggling of charged particles, the energy loss in the target, and the divergence and energy spread of the beam. The effects of the telescope resolution and efficiency were included, along with the absorption of neutrons by the telescope [a $10 \%$ effect [25] ]. The momentum spread arising from the neutron removal was determined from the measured angular distribution of neutrons from the very low energy decay of ${ }^{11} \mathrm{Be}^{*}(4.0 \mathrm{MeV})$ to ${ }^{10} \mathrm{Be}^{*}\left(2^{+}, 3.37 \mathrm{MeV}\right)+n$ (cf. Fig. 2), where the neutron momentum distribution was dominated by that of the ${ }^{11} \mathrm{Be}^{*}$ before decay. The detection of a fast neutron $\left(E_{n}>15 \mathrm{MeV}\right)$ from the breakup of ${ }^{12} \mathrm{Be}$, measured in coincidence with a ${ }^{10} \mathrm{Be}$ from the subsequent decay of ${ }^{11} \mathrm{Be}^{*}$, was also simulated.

Simulations were performed for the decay of the unbound states in ${ }^{11} \mathrm{Be}$ below $4 \mathrm{MeV}$ (assumed isotropic in the ${ }^{11} \mathrm{Be}$ rest frame), including the decay from the $\sim 4 \mathrm{MeV}$ state to the $2^{+}$state in ${ }^{10} \mathrm{Be}$, and also the detection of neutrons diffracted from ${ }^{12} \mathrm{Be}$ in coincidence with a

${ }^{10} \mathrm{Be}$ core. The simulated events were analyzed in the same 
TABLE I. Cross sections for states in ${ }^{11} \mathrm{Be}$ produced via neutron removal from ${ }^{12} \mathrm{Be}$ on a carbon target at $39.3 \mathrm{MeV} / \mathrm{nucleon}$ (present work) compared with reaction calculations and previous work at $78 \mathrm{MeV} /$ nucleon on ${ }^{9}$ Be. Uncertainties for $S_{\text {exp }}$ are experimental only [for comparison, Ref. [3] values have been adjusted to remove the assumed $20 \%$ theory uncertainty].

\begin{tabular}{|c|c|c|c|c|c|c|c|c|c|c|}
\hline$J^{\pi}$ & $\begin{array}{c}E_{x} \\
(\mathrm{MeV})\end{array}$ & $\begin{array}{l}\sigma_{\exp } \\
(\mathrm{mb})\end{array}$ & $\begin{array}{l}\sigma_{\text {strip }} \\
(\mathrm{mb})\end{array}$ & $\begin{array}{l}\sigma_{\text {diff }} \\
(\mathrm{mb})\end{array}$ & $\begin{array}{c}\sigma_{\mathrm{sp}} \\
(\mathrm{mb})\end{array}$ & $\begin{array}{c}S_{\exp }{ }^{\mathrm{a}} \\
\text { (present work) }\end{array}$ & $\begin{array}{l}S_{\text {exp }}{ }^{\text {a }} \\
\text { Ref. [3] }\end{array}$ & $\begin{array}{l}\text { WBT2 } \\
\text { Ref. [3] }\end{array}$ & $\begin{array}{l}\mathrm{WBT}^{\prime} \\
\text { (see text) }\end{array}$ & $\begin{array}{c}\text { EXC2 } \\
\text { Ref. [14] }\end{array}$ \\
\hline $1 / 2^{+}$ & 0.00 & $\mathrm{~b}$ & 61.28 & 57.69 & 118.96 & {$[0.56 \pm 0.18]^{\mathrm{b}}$} & $0.42 \pm 0.05$ & 0.69 & 0.55 & 0.44 \\
\hline $1 / 2^{-}$ & 0.32 & $32.5 \pm 6.1$ & 42.59 & 30.72 & 73.31 & $0.44 \pm 0.08$ & $0.37 \pm 0.07$ & 0.58 & 0.47 & 0.38 \\
\hline $5 / 2^{+}$ & 1.78 & $30.3 \pm 4.0$ & 39.78 & 22.77 & 62.55 & $0.48 \pm 0.06$ & $\cdots$ & 0.55 & 0.44 & 0.58 \\
\hline $3 / 2^{-}$ & 2.69 & $22.6 \pm 4.1$ & 35.47 & 20.87 & 56.35 & $0.40 \pm 0.07$ & $\cdots$ & & & \\
\hline
\end{tabular}

${ }^{a}$ Uncertainties do not include contribution from theoretical model of reaction mechanism, estimated to be $\pm 10 \%-20 \%$.

${ }^{\mathrm{b}}$ Total $\sigma_{\text {exp }}$ not measured, but $\sigma_{\text {diff }}=46 \pm 10 \mathrm{mb}$ for $1 / 2^{+}$and $1 / 2^{-}$together and $S_{\text {exp }}$ deduced from this (see text).

manner as the experimental data. The resulting $E_{\text {rel }}$ line shapes (Fig. 2) were least-squares fitted to the experimental distribution and, using the detection efficiency determined from the simulations (Fig. 2, upper panel), the cross sections for the different states were determined. As a consistency check, the ${ }^{11} \mathrm{Be}$ transverse momentum distribution (from ${ }^{10} \mathrm{Be}+n$ ), and the neutron angular distribution $\frac{d \sigma_{n}}{d \Omega}$ in coincidence with ${ }^{10} \mathrm{Be}$, were reconstructed from the simulated events. In both cases the agreement with experiment was very good. The simulated "hit" efficiency for neutrons agreed with the value determined by fitting and integrating $\frac{d \sigma_{n}}{d \Omega}$ to within $\sim 1 \%$.

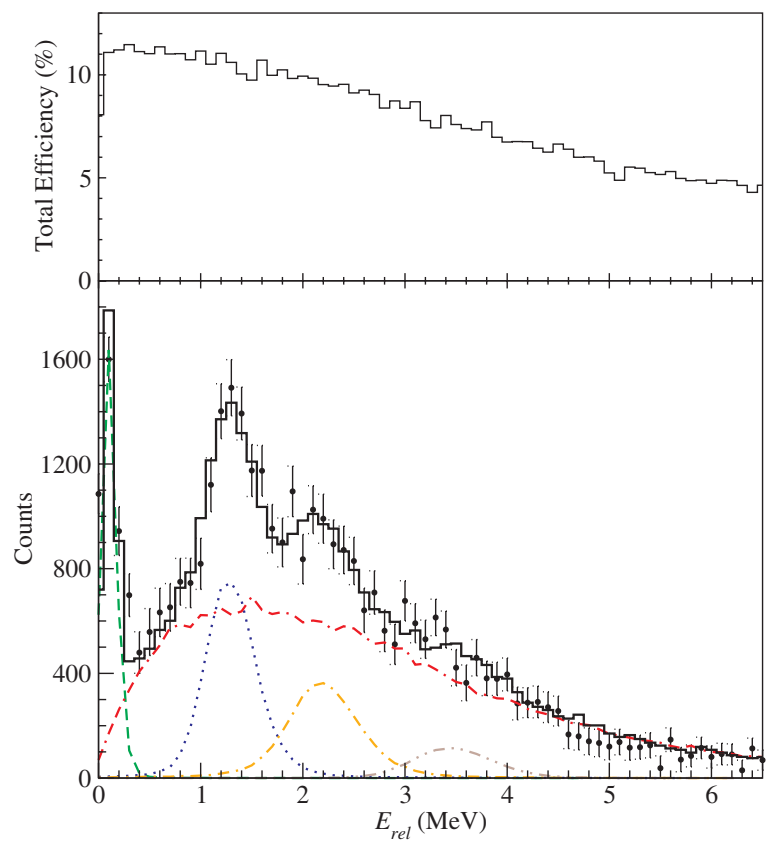

FIG. 2 (color online). Relative energy spectrum of ${ }^{10} \mathrm{Be}+n$ (lower panel) where the solid points represent the experimental data. The histogram depicts the result of the full simulation; the line shapes of the individual components of the simulation are shown (see text). The $1.78 \mathrm{MeV}$ state in ${ }^{11} \mathrm{Be}$ is clearly visible at $E_{\text {rel }} \approx 1.3 \mathrm{MeV}\left(S_{\mathrm{n}}=0.50 \mathrm{MeV}\right)$. The resolution in $E_{\text {rel }}$ varies as $a_{0} E^{1 / 2}$ (where $a_{0}$ is a constant), and at $1 \mathrm{MeV}$ is $\sim 400 \mathrm{keV}$ (FWHM). The upper panel depicts the simulated array efficiency.
The cross section extracted for the production of the $1.78 \mathrm{MeV} 5 / 2^{+}$state is included in Table I, along with that for the $2.69 \mathrm{MeV} 3 / 2^{-}$state and the bound $1 / 2^{-}$state. The estimated uncertainties include contributions from statistical fitting plus uncertainties in the efficiency corrections, target thickness, and background subtraction. The cross section for diffractive breakup to produce a bound ${ }^{11} \mathrm{Be}$ (either $1 / 2^{+}$or $1 / 2^{-}$) and a fast neutron was also extracted and was $46 \pm 10 \mathrm{mb}$.

The measured neutron removal partial cross sections from ${ }^{12} \mathrm{Be}$ can be interpreted in terms of spectroscopic factors $\left(S_{\text {exp }}\right)$ using a reaction model. The $S_{\text {exp }}$ listed in Table I are, except for the $1 / 2^{+}$state, the ratio of the experimental $\left(\sigma_{\exp }\right)$ to the eikonal model partial cross section $\left(\sigma_{\mathrm{sp}}\right)$. For the $1 / 2^{+}$state, the measurement of diffractive breakup of ${ }^{12} \mathrm{Be}$ to the two bound ${ }^{11} \mathrm{Be}$ states has been used, with the $1 / 2^{-}$contribution being subtracted according to its theoretical diffraction cross section (see Table I) and the $S_{\exp }$ of $0.44 \pm 0.08$ found here. The uncertainties quoted for $S_{\text {exp }}$ in Table I do not include contributions from the assumptions in the reaction calculation, where, for example, two-step processes are not included, and it is estimated that this implies an additional uncertainty of up to $20 \%$ [consistent with Ref. [3] ].

The present reaction analysis follows closely the eikonal model of Refs. [3,26]. The neutron-target $S$ matrix was computed from the target density and the JLM effective nucleon-nucleon interaction [27]. The usual real and imaginary part scale factors $\left(\lambda_{V}=1.0, \lambda_{W}=0.8\right)$ were applied to the optical potential. The matter densities for ${ }^{12} \mathrm{C}$ and ${ }^{10} \mathrm{Be}$ were of harmonic oscillator and Gaussian form, respectively, with rms radii of 2.4 and $2.28 \mathrm{fm}$. For the $1 / 2^{+}, 1 / 2^{-}$, and $5 / 2^{+}$(particle) transitions, $\left({ }^{10} \mathrm{Be}+n\right.$ ) composite core-target $S$ matrices were constructed from those of ${ }^{10} \mathrm{Be}$ and the neutron [26]. In the (unbound) $5 / 2^{+}$ case, a separation energy of $0.01 \mathrm{MeV}$ was used to compute the $S$ matrix. For the $3 / 2^{-}$(hole) state, a Gaussian (mass 11) core density of rms radius $2.54 \mathrm{fm}$ was used, representative of the size of ${ }^{12} \mathrm{Be}$. The removed-nucleon single-particle overlaps were taken as eigenstates of Woods-Saxon potentials, with geometry $r_{0}=1.25 \mathrm{fm}$ and $a=0.7 \mathrm{fm}$, and with depths adjusted to the physical separation energies for each final state. 
Our observation of the $5 / 2^{+}$state in knockout from ${ }^{12} \mathrm{Be}$ is the first direct experimental evidence for a significant $d$-wave intruder component in any $N=8$ isotone. Barker [6] first pointed out that the observed lowering of the $1 s_{1 / 2}$ and $0 d_{5 / 2}$ neutron orbitals in ${ }^{11} \mathrm{Be}$ should lead to strong admixtures of these orbitals in the ground state of ${ }^{12} \mathrm{Be}$ and concluded [6,7] that as little as $20 \%-40 \%$ of the ${ }^{12} \mathrm{Be}$ ground state might comprise the $0 \hbar \omega \nu\left(0 p_{1 / 2}\right)^{2}$ configuration. This model also successfully predicted a strong $1 s_{1 / 2}$ admixture in the ${ }^{11} \mathrm{Li}$ ground state.

The $(1 s 0 d)^{2}$ contribution to the ${ }^{12} \mathrm{Be}$ ground state was deduced in an indirect fashion by Fortune et al. [9] in light of their measurements of $\tau_{1 / 2}$ and $E\left(2_{1}^{+}\right)$for ${ }^{12} \mathrm{Be}[8,28]$. Indeed, according to subsequent $p s d$-space shell-model calculations, the $\beta$ decay from the ${ }^{12} \mathrm{Be}$ ground state is quenched to an extent that places an upper limit of $35 \%$ on the $0 \hbar \omega$ component of the wave function [29]. The ${ }^{12} \mathrm{O}-{ }^{12} \mathrm{Be}$ Coulomb energy difference also supports shell breaking of this order [30].

The magnitude of shell breaking observed in the present work can be quantified by comparing with theory. Table I includes spectroscopic factors (WBT2) based on the shellmodel calculations reported in Ref. [3], which correspond to a mixing of $32 \% 0 \hbar \omega\left(0 p^{8}\right)$ and $68 \% 2 \hbar \omega\left(0 p^{6}(1 s 0 d)^{2}\right)$ contributions. The sum of these is close to the value 2.0 that would be expected in the simplest picture. These values are scaled by 0.8 to give WBT2' [which is consistent with other knockout work [31]] and this reproduces the present experimental results very well. Also listed for comparison, the EXC2 values from a 3-body model including ${ }^{10} \mathrm{Be}$ core excitation [14] give good agreement with the present work while reproducing other features of ${ }^{12} \mathrm{Be}$.

Recently, Iwasaki et al. inferred the disappearance of the $N=8$ shell gap in ${ }^{12} \mathrm{Be}$ from both the deformation length derived from inelastic proton scattering to the $2_{1}^{+}$state [2], and the low energy and large $B\left(E 1 ; 0^{+} \rightarrow 1^{-}\right)$for the $1_{1}^{-}$ state [4]. Their deductions agreed in detail with the $p s d$ shell-model calculations $[13,29]$, concluding that the $0 p_{1 / 2}$ and $1 s_{1 / 2}$ orbitals are effectively degenerate for ${ }^{12} \mathrm{Be}$, just as they are in ${ }^{11} \mathrm{Be}$.

Consistent theoretical results are obtained using nuclear field theory [12], which predicts both the presence of large $\nu\left(0 d_{5 / 2}\right)^{2}$ strength in ${ }^{12} \mathrm{Be}$ and its absence in ${ }^{11} \mathrm{Li}$, in agreement with a recent theoretical analysis [32] of ${ }^{11} \mathrm{Li}$ reaction data [1]. It is interesting to note that the early work of Barker [6] also predicted a low-lying $0_{2}^{+}$state at $2.35 \mathrm{MeV}$ in ${ }^{12} \mathrm{Be}$ which has only recently been observed, at $2.24 \mathrm{MeV}$ [5].

Thus, the neutron shell breaking observed at $N=8$ for ${ }^{12} \mathrm{Be}$, but not ${ }^{14} \mathrm{C}$, is similar to the breaking of $N=20$ for ${ }^{32} \mathrm{Mg}$ but not ${ }^{34} \mathrm{Si}$. In each case, when the proton $j_{>}$orbital is full, the neutrons are magic. When protons are removed, the full $j_{<}$orbital for neutrons is no longer magic, the next shell intrudes, and deformation results.
In conclusion, single-neutron removal cross sections from ${ }^{12} \mathrm{Be}$ to the $0.32 \mathrm{MeV}\left(1 / 2^{-}\right)$and $1.78 \mathrm{MeV}\left(5 / 2^{+}\right)$ states in ${ }^{11} \mathrm{Be}$ have been measured. From these and eikonal model calculations, spectroscopic factors were deduced. These indicate strong breaking of the $N=8$ magic number in ${ }^{12} \mathrm{Be}$, including a significant $d$-wave component. This is distinct from other $N=8$ isotones and is the first direct experimental confirmation of the predictions of a number of structure models.

The authors wish to acknowledge the support provided by the technical staff of LPC and GANIL. Partial support through the EU Human Mobility programme of the European Community is also acknowledged.

*Present address: Rutgers University, c/o Physics Division, Oak Ridge National Laboratory, TN 37831-6354, USA.

†Present address: Rudjer Bošković Institute, Bijenička 54, HR-10000 Zagreb, Croatia.

[1] H. Simon et al., Phys. Rev. Lett. 83, 496 (1999).

[2] H. Iwasaki et al., Phys. Lett. B 481, 7 (2000).

[3] A. Navin et al., Phys. Rev. Lett. 85, 266 (2000).

[4] H. Iwasaki et al., Phys. Lett. B 491, 8 (2000).

[5] S. Shimoura et al., Phys. Lett. B 560, 31 (2003).

[6] F. C. Barker, J. Phys. G 2, L45 (1976).

[7] F. C. Barker and G. T. Hickey, J. Phys. G 3, L23 (1977).

[8] D. E. Alburger et al., Phys. Rev. C 18, 2727 (1978).

[9] H. T. Fortune et al., Phys. Rev. C 50, 1355 (1994).

[10] B. A. Brown, Prog. Part. Nucl. Phys. 47, 517 (2001).

[11] Y. Kanada-En'yo and H. Horiuchi, Phys. Rev. C 68, 014319 (2003).

[12] G. Gori et al., Phys. Rev. C 69, 041302(R) (2004).

[13] H. Sagawa et al., Phys. Rev. C 63, 034310 (2001).

[14] F. Nunes et al., Nucl. Phys. A703, 593 (2002).

[15] J.C. Pacheco and N. Vinh Mau, Phys. Rev. C 65, 044004 (2002).

[16] B. Zwieglinski et al., Nucl. Phys. A315, 124 (1979).

[17] N. Fukuda et al., Phys. Rev. C 70, 054606 (2004).

[18] F. Ajzenberg-Selove, Nucl. Phys. A506, 1 (1990).

[19] S. Ahmed et al., Phys. Rev. C 69, 024303 (2004).

[20] I. Tilquin et al., Nucl. Instrum. Methods Phys. Res., Sect. A 365, 446 (1995).

[21] M. Labiche et al., Phys. Rev. Lett. 86, 600 (2001).

[22] S. D. Pain, Ph.D. thesis, University of Surrey, 2004.

[23] D. J. Millener, Nucl. Phys. A693, 394 (2001).

[24] H. G. Bohlen et al., Nucl. Phys. A734, 345 (2004).

[25] J. L. Lecouey, Ph.D. thesis, Université de Caen, 2003.

[26] J. A. Tostevin, Nucl. Phys. A682, 320c (2001).

[27] J. P. Jeukenne, A. Lejeune, and C. Mahaux, Phys. Rev. C 16, 80 (1977).

[28] D. E. Alburger et al., Phys. Rev. C 17, 1525 (1978).

[29] T. Suzuki and T. Otsuka, Phys. Rev. C 56, 847 (1997).

[30] R. Sherr and H. T. Fortune, Phys. Rev. C 60, 064323 (1999).

[31] P. G. Hansen and J. A. Tostevin, Annu. Rev. Nucl. Part. Sci. 53, 219 (2003).

[32] C. A. Bertulani and P. G. Hansen, Phys. Rev. C 70, 034609 (2004). 